\title{
Predicting postoperative total calcium require- ments after parathyroidectomy in secondary hyperparathyroidism
}

Byung Heon Kang ${ }^{1}$, Soon Young Hwang ${ }^{2}$, Jeong Yeop Kim ${ }^{1}$, Yu Ah Hongㄹ, Mi Yeon Jung ${ }^{1}$, Eun Ah Lee Ji Eun Lee ${ }^{3, *}$, Jae Bok Lee ${ }^{4}$, Gang Jee Ko ${ }^{1}$, Heui Jung $\mathrm{Pyo}^{1}$, and Young Joo Kwon ${ }^{1}$

\author{
${ }^{1}$ Division of Nephrology, \\ Department of Internal Medicine, \\ ${ }^{2}$ Department of Biostatistics, \\ Korea University College of \\ Medicine, Seoul; ${ }^{3}$ Division \\ of Nephrology, Department \\ of Internal Medicine, Hallym \\ University College of Medicine, \\ Chuncheon; ${ }^{4}$ Division of \\ Breast and Endocrine Surgery, \\ Department of Surgery, Korea \\ University College of Medicine, \\ Seoul, Korea
}

Received: July 19, 2013

Revised : August 27, 2013

Accepted: May 11, 2014

\section{Correspondence to}

Young Joo Kwon, M.D.

Department of Internal Medicine, Korea University Guro Hospital, 148 Gurodong-ro, Guro-gu, Seoul o8308, Korea Tel: +82-2-2626-3036

Fax: +82-2-2626-2024

E-mail: yjkwon@korea.ac.kr

*Current affiliation: Division of Nephrology, Department of Internal Medicine, Wonkwang University Sanbon Hospital, Gunpo, Korea
Background/Aims: To prevent hypocalcemia after parathyroidectomy (PTX), parenteral calcium is required in addition to oral calcitriol and calcium. After switching to oral calcium, patients can be discharged from the hospital. The aim of this study was to analyze the clinical characteristics and outcomes of PTX performed at a single Korean center and to investigate the associated laboratory factors used to analyze the total amount of postoperative calcium required.

Methods: We enrolled 91 hemodialysis patients undergoing PTX from November 2003 to December 2011. We collected clinical and laboratory data preoperatively, 12 and 48 hours postoperatively, at discharge, and 3 and 6 months postoperatively. Results: In total, 59 patients underwent PTX with autotransplantation (AT), 6 underwent total PTX without AT, 11 underwent subtotal PTX, and 15 underwent limited PTX. Total PTX without AT showed the lowest recurrence rate. At all postoperative time points, the mean levels of serum calcium, phosphorus, and intact parathyroid hormone (iPTH) decreased significantly, compared with preoperative levels; however, alkaline phosphatase (ALP) increased significantly from 48 hours postoperatively to discharge $(p<0.001)$. On multiple linear regression analysis, the total amount of injected calcium during hospitalization showed a significant correlation with preoperative ALP $(p<0.001)$, preoperative iPTH $(p=$ 0.037), and $\Delta$ phosphorus at 48 hours $(p<0.001)$. We developed an equation for estimating the total calcium requirement after PTX.

Conclusions: Preoperative ALP, preoperative iPTH, and $\Delta$ phosphorus at 48 hours may be significant factors in estimating the postoperative calcium requirement. The formula for postoperative calcium requirement after PTX may help to predict the duration of postoperative hospitalization.

Keywords: Hyperparathyroidism; Secondary; Parathyroidectomy; Kidney failure, chronic

\section{INTRODUCTION}

Secondary hyperparathyroidism is one of the most common complications in patients with chronic kidney dis- ease (CKD), including those on maintenance hemodialysis (HD). CKD-mineral bone disorder (MBD) includes (1) imbalances in calcium and phosphate homeostasis, active vitamin $\mathrm{D}$ deficiency, and increased levels of in- 
tact parathyroid hormone (iPTH), (2) skeletal deformities, and (3) extraskeletal calcification [1,2].

The management of CKD-MBD includes normalization of serum phosphorus and serum calcium levels, optimization of the iPTH level, and improvement in skeletal deformities by medical therapy, such as phosphate binders, active vitamin $\mathrm{D}$, and calcimimetics [3-6]. Despite medical therapy, $10 \%$ to $30 \%$ of end-stage renal disease (ESRD) patients who undergo HD for more than 10 years require a parathyroidectomy (PTX) $[7,8]$. PTX is an available treatment modality in cases of unresponsiveness to medical therapy. Successful PTX can have a striking effect in reducing serum levels of calcium, phosphate, iPTH, and alkaline phosphatase (ALP). In some cases, postoperative hypocalcemia is severe and prolonged despite normal or even elevated levels of iPTH. The abrupt withdrawal of PTH results in a transient marked increase in bone remineralization and a rapid shift of calcium from the circulation to bone tissue $[5,9]$. This phenomenon, called 'hungry bone syndrome,' occurs most often in patients who have developed bone disease preoperatively due to a chronic increase in bone resorption, induced by high levels of PTH [7-9].

Hypocalcemia remains a frequently encountered complication, and its prevention represents a clinical challenge because pronounced hypocalcemia is very serious. The maintenance of a stable postoperative serum calcium level is critical in preventing hungry bone syndrome [10]. Prediction of the total postoperative calcium requirement would be of interest to determine the postoperative hospitalization period. A previous study suggested that ALP could be a useful parameter in determining the amount of calcium supplementation required after PTX [11]; however, there is no previous report for estimating the total postoperative calcium requirement after PTX in patients with secondary hyperparathyroidism.

In the present study, we aimed to analyze the clinical characteristics and outcomes of PTX in a single center and to investigate clinical factors that may be related to the postoperative calcium requirement. We focused on developing an equation that could estimate the total calcium requirement after PTX.

\section{METHODS}

This study included 91 patients who underwent PTX because of secondary hyperparathyroidism between November 2003 and December 2011 at Korea University Guro Hospital. The research protocol was approved by the Institutional Review Board of Korea University.

In total, 94 PTX were performed, including two cases of persistent hyperparathyroidism and one case of recurrent hyperparathyroidism. Clinical and biochemical data were collected from electronic medical records. Clinical data included gender, age, body weight, presence of diabetes mellitus, duration of dialysis, mode of renal replacement therapy (HD or peritoneal dialysis [PD]), systolic and diastolic blood pressure, indication for PTX, mode of anesthesia (general anesthesia [GA] or local anesthesia [LA]), type of operation (total PTX with autotransplantation [AT], total PTX without AT, subtotal PTX, limited PTX), pathology of the parathyroid glands, postoperative complications, hospitalization duration, and recurrence rate.

Preoperative localization studies were performed in all patients. Patients who had a single adenoma strongly suspected on preoperative localization studies underwent limited PTX, and patients with multi-gland disease or those who had failed preoperative localization underwent total PTX with or without AT or subtotal PTX. A simultaneous thymectomy was not performed in any patient.

Indications for the type of PTX were similar to other studies [8] and included the following: (1) high level of parathyroid hormone (iPTH $>500 \mathrm{pg} / \mathrm{mL}$ ); (2) detection of enlarged parathyroid glands by image diagnosis (the longest length of at least one gland $>1 \mathrm{~cm}$ on computed tomography); and (3) uncontrolled hypercalcemia and hyperphosphatemia or multiple severe bone deformities or extraskeletal calcifications when the iPTH level was $<500 \mathrm{pg} / \mathrm{mL}$.

The number of parathyroid glands excised, the longest parathyroid gland length, the heaviest parathyroid gland weight measured, and the histopathological diagnoses of parathyroid tissues were collected. Recurrence of secondary hyperparathyroidism was defined as an iPTH $>300 \mathrm{pg} / \mathrm{mL}$ at 6 months postoperatively. Laboratory parameters were evaluated in all patients from medical records preoperatively and at multiple time points 
postoperatively. Biochemical data, including calcium, ionized calcium, phosphorus, iPTH, ALP, albumin, and hemoglobin, were collected preoperatively and 12 and 48 hours postoperatively, at the time of discharge, and 3 and 6 months postoperatively. Preoperative data were collected at the preoperative assessment 1 to 2 days prior to surgery. iPTH levels were measured using radioimmunoassay kits (Cisbio Bioassay, Codolet, France).

Postoperative calcium supplements in the form of intravenous calcium gluconate, oral calcium carbonate, and oral calcium lactate with $2 \mu \mathrm{g}$ of oral calcitriol were prescribed, per our standard protocol. In the initial standard protocol, oral calcium carbonate (equivalent to $200 \mathrm{mg}$ elemental calcium per $500 \mathrm{mg}$ tablet) was started at a dose of 1,000 mg three times daily and intravenous calcium gluconate was given daily and titrated based on serum calcium and ionized calcium levels while the infusion was titrated down. Calcium lactate was added if the tablet burden of calcium carbonate was high. The total calcium amount during the hospitalization period after PTX was totaled and converted to mEq.

\section{Statistical analysis}

Statistical analyses were performed with the SPSS version 12.0.1 (SPSS Inc., Chicago, IL, USA), and all variables are presented as mean $\pm \mathrm{SD}$ or median (range). To evaluate changes in biochemical parameters of mineral bone disease after PTX, a paired t test was performed, pairing preoperative and postoperative data. For variables that were not normally distributed, a logarithmic transformation was used to make the calcium injection values to fit a normal distribution. Pearson correlation coefficients and univariate linear regression were used to examine associations between injected calcium and laboratory parameters associated with mineral bone disease. Among significant factors, a multiple linear regression analysis was performed to develop an equation for the predicted total elemental calcium requirement. Values of $p<0.05$ were considered to indicate statistical significance.

\section{RESULTS}

In total, 91 patients with secondary hyperparathyroidism underwent PTX between 2003 and 2011. In total, 94 PTX were performed due to recurrent hyperparathyroidism. Demographic data of the study population are shown in Table 1. The mean age was 48.9 years (range, 19.9 to 70 ), and the mean duration of dialysis was 12.8 years (range, 1 to 27). Most patients were undergoing HD, although 11 patients $(12 \%)$ were on continuous ambulatory PD. The mean duration of hospitalization was $18.64 \pm 13.17$ days

Table 1. Demographics and clinical characteristics of the study population $(n=91$, persistence 2 , recurrence 1$)$

\begin{tabular}{|c|c|}
\hline Characteristic & Value \\
\hline Age, yr & $48.9 \pm 10.3$ \\
\hline Male sex & $46(50.5)$ \\
\hline Body weight, kg & $58.1 \pm 10.4$ \\
\hline Dialysis duration, yr & $12.8 \pm 5.6$ \\
\hline Hemodialysis & $80(87.9)$ \\
\hline Diabetes mellitus & $8(8.8)$ \\
\hline \multicolumn{2}{|l|}{ Blood pressure } \\
\hline Systolic blood pressure, $\mathrm{mmHg}$ & $131.7 \pm 18.3$ \\
\hline Diastolic blood pressure, mmHg & $78.7 \pm 10.1$ \\
\hline Bone mineral density score & $-2.9 \pm 1.7$ \\
\hline \multicolumn{2}{|l|}{ Preoperative laboratory data } \\
\hline $\mathrm{Ca}, \mathrm{mg} / \mathrm{dL}$ & $10.6 \pm 1.3$ \\
\hline Ionized calcium, mg/dL & $5.0 \pm 0.5$ \\
\hline Phosphorus, mg/dL & $5.9 \pm 1.7$ \\
\hline ALP, IU/L (reference value, 8-76) & $313.6 \pm 308.1$ \\
\hline Albumin, g/dL & $4.1 \pm 0.8$ \\
\hline Parathyroid hormone, pg/mL & $1,385 \cdot 5(154 \cdot 6-1,742 \cdot 4)$ \\
\hline Hemoglobin, g/dL & $11.0 \pm 1.6$ \\
\hline \multicolumn{2}{|l|}{ Phosphate binders $(n=94)$} \\
\hline Al containing phosphate binders & $10(10.6)$ \\
\hline Ca containing phosphate binders & $28(29.8)$ \\
\hline Sevelamer & $26(27 \cdot 7)$ \\
\hline Lanthanum & $19(20.2)$ \\
\hline None & $11(11.7)$ \\
\hline \multicolumn{2}{|l|}{ Vitamin D $(\mathrm{n}=94)$} \\
\hline Cholecalciferol & $3(3.2)$ \\
\hline Calcitriol & $29(30.9)$ \\
\hline Pericalcitol & $24(25 \cdot 5)$ \\
\hline Cinacalcet & $6(6.4)$ \\
\hline None & $32(34.0)$ \\
\hline
\end{tabular}

Values are presented as mean $\pm \mathrm{SD}$, number (\%), or median (range).

ALP, alkaline phosphatase. 
(range, 2 to 74 ). In 88 patients, the indication for PTX was uncontrolled secondary hyperparathyroidism despite medical therapy. The most common symptoms associated with secondary hyperparathyroidism included musculoskeletal aches, fatigue, and pruritus. The symptoms did not correlate with biochemical markers. The mean level of preoperative iPTH was 1,647.1 $\pm 1,803.1 \mathrm{pg} /$ $\mathrm{mL}$ (median, 1,385.5; range, 544.6 to $1,742.4$ ) with an enlarged parathyroid gland. In the other six cases, where PTX was indicated due to uncontrolled hypercalcemia (serum calcium level > $12 \mathrm{mg} / \mathrm{dL}$ ), the iPTH level was < $500 \mathrm{pg} / \mathrm{mL}$.

Two modes of anesthesia were used: GA and LA. In 94 surgeries, 11 cases (12\%) were performed under LA due to poor preoperative general condition. Four types of PTX were performed: total PTX with AT, total PTX without AT, subtotal PTX, and limited PTX. There were 59 (63\%) total PTX with AT and 6 (6\%) total PTX without AT. It was not possible to transplant AT to the patients' forearms because AT was contraindicated according to glandular pathological findings. There were 11 (12\%) subtotal PTXs in patients awaiting kidney transplantation or patients with an aluminum pre-exposure history. There were 15 (10\%) limited PTX due to a single parathyroid adenoma or an ectopic parathyroid gland.

In 94 operations, the length of the longest excised parathyroid glands was measured in 93 cases, and 58 glands were weighed. The mean length of the longest gland was $2.4 \pm 0.8 \mathrm{~cm}$ (range, 0.5 to 5.0 ), and the mean weight of the heaviest gland was $2.7 \pm 2.5 \mathrm{~g}$ (range, 0.28 to 10). The distribution of the final histopathological diagnoses for the excised parathyroid glands is shown in Fig. 1; diffuse hyperplasia (45 cases, $47.87 \%$ ) and nodular hyperplasia (45 cases, $47.87 \%$ ) showed the highest incidence in the excised parathyroid glands. Parathyroid adenoma and carcinoma were identified in three cases (3.19\%) and one cases (1.1\%), respectively.

In this study, there were few complications after PTX. The main postoperative complication associated with PTX was transient hoarseness (5\%). There was one patient with persistent hoarseness after 6 months. No postoperative bleeding requiring surgical intervention was noted. One patient died within 3 months after PTX due cardiovascular complications, not postoperative complications. There were 19 cases of recurrence, defined as an $\mathrm{iPTH}>300 \mathrm{pg} / \mathrm{mL}$ at 6 months after PTX. Conse-

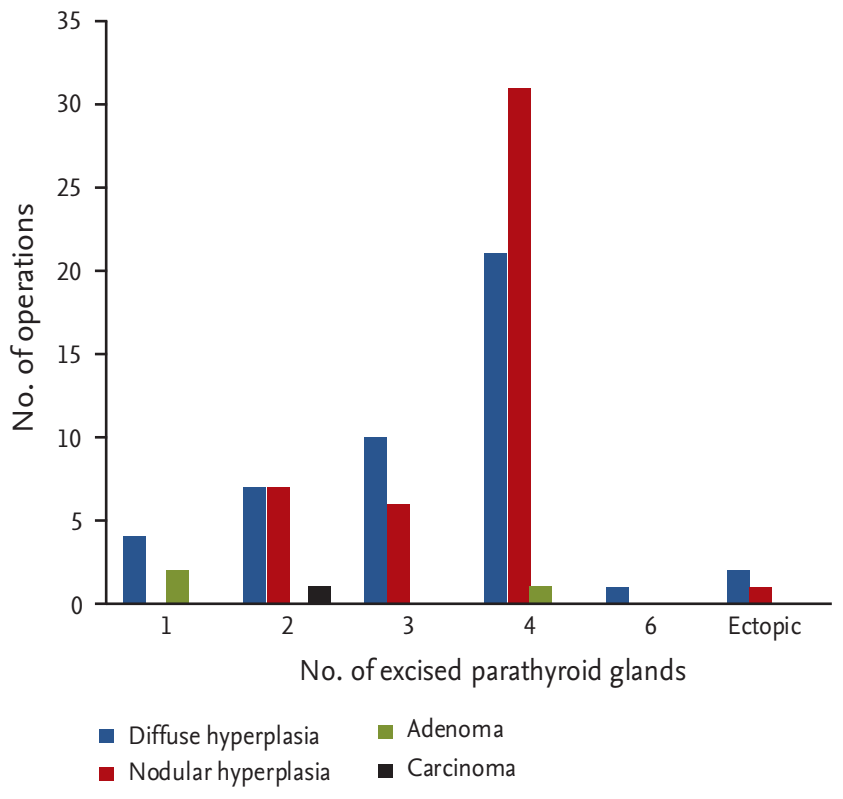

Figure 1. Histopathological profiles of resected parathyroid glands.

quently, the recurrence rate after PTX in our center was $20.2 \%$. Among these patients, four were able to maintain iPTH levels $<300 \mathrm{pg} / \mathrm{mL}$ at the 1-year follow-up.

At each of the five postoperative time points (12 hours postoperatively, 48 hours postoperatively, discharge, 3 months postoperatively, 6 months postoperatively), the mean levels of serum calcium, phosphorus, and iPTH decreased, while ALP levels increased, compared with preoperative levels. Serum calcium, phosphorus, and iPTH showed statistical differences between preoperative and postoperative data at each time point (Fig. 2). PTH levels decreased significantly within 12 hours after PTX (preoperative iPTH 1,647.08 $\pm 1,803.15 \mathrm{pg} / \mathrm{mL}$ vs. postoperative 12 hours iPTH $299.14 \pm 577.99 \mathrm{pg} / \mathrm{mL}, p=$ o.016). Postoperative PTH levels remained in the optimal target range, according to Kidney Disease Outcomes Quality Initiative guidelines ( 150 to $300 \mathrm{pg} / \mathrm{mL}$ ) from 12 hours to 6 months after PTX. Analogous to the course of PTH levels, serum calcium and phosphorus levels decreased dramatically within 12 hours after PTX. Mean calcium $(9.28 \pm 1.18 \mathrm{mg} / \mathrm{dL})$ and phosphorus $(4.79 \pm 1.52$ $\mathrm{mg} / \mathrm{dL}$ ) remained at target levels (serum calcium < 9.5 $\mathrm{mg} / \mathrm{dL}$, serum phosphorus $<5.5 \mathrm{mg} / \mathrm{dL}$ ) within 6 months postoperatively. Serum calcium and phosphorus levels postoperatively were significantly lower compared with preoperative serum calcium and phosphorous levels 
(preoperative vs. 6 months postoperative calcium, 10.61 $\pm 1.31 \mathrm{mg} / \mathrm{dL}$ vs. $9.27 \pm 1.18 \mathrm{mg} / \mathrm{dL}, p<0.0001$; preoperative vs. 6 months postoperative phosphorus, $5.89 \pm 1.74$ $\mathrm{mg} / \mathrm{dL}$ vs. $4.79 \pm 1.51 \mathrm{mg} / \mathrm{dL}, p=0.003)$. Mean ALP (456 \pm 454.67 IU/L; range, 49 to 3,037) was increased significantly at discharge $(p<0.001)$ but returned to the normal range by 6 months postoperatively (Fig. 2). Transient hypocalcemia, defined as a serum calcium level $<8.5 \mathrm{mg} / \mathrm{dL}$, was observed in $71 \%$ of patients. Severe hypocalcemia, defined as a serum calcium level $<7.5 \mathrm{mg} / \mathrm{dL}$, was observed in $25 \%$ of patients. Calcium was administered parenterally in the immediate postoperative period and later administered orally via tablets with calcitriol to prevent postoperative hypocalcemia. All patients were given an oral calcium supplement and calcitriol at discharge. The total amounts of injected calcium 24, 48 hours, and 7 days after PTX, and during the total hospitalization period, were 61.03 $\pm 44.16,135.76 \pm 78.96,502.73 \pm 262.98$, and 1,049.1 $\pm 936.6 \mathrm{mEq}$, respectively (Fig. 3).

The duration of postoperative hospitalization cor- related significantly with the total amount of calcium supplement $(r=0.775, p<0.001)$. A longer length of hospitalization could itself be a cause of increased morbidity and potentially be related to adverse outcomes. Univariate analysis was performed to clarify the correlation between preoperative laboratory parameters and changes in calcium and phosphorus in the postoperative state and $\log$ (total calcium injected during hospitalization). Log (total calcium injected during hospitalization) correlated with preoperative ALP, preoperative iPTH, $\Delta$ phosphorus at 48 hours, and $\Delta$ calcium at 12 hours in univariate analyses (Table 2). However, in a multiple linear regression analysis, log (total calcium injected during hospitalization) correlated with preoperative $\operatorname{ALP}(p<0.001)$, preoperative iPTH $(p=0.037)$, and $\Delta$ phosphorus at 48 hours $(p<0.001)$, but not $\Delta$ calcium at 12 hours. Using the variables with significant relationships in the multiple linear regression analysis, the following equation was developed for estimating the total calcium requirement during hospitalization:
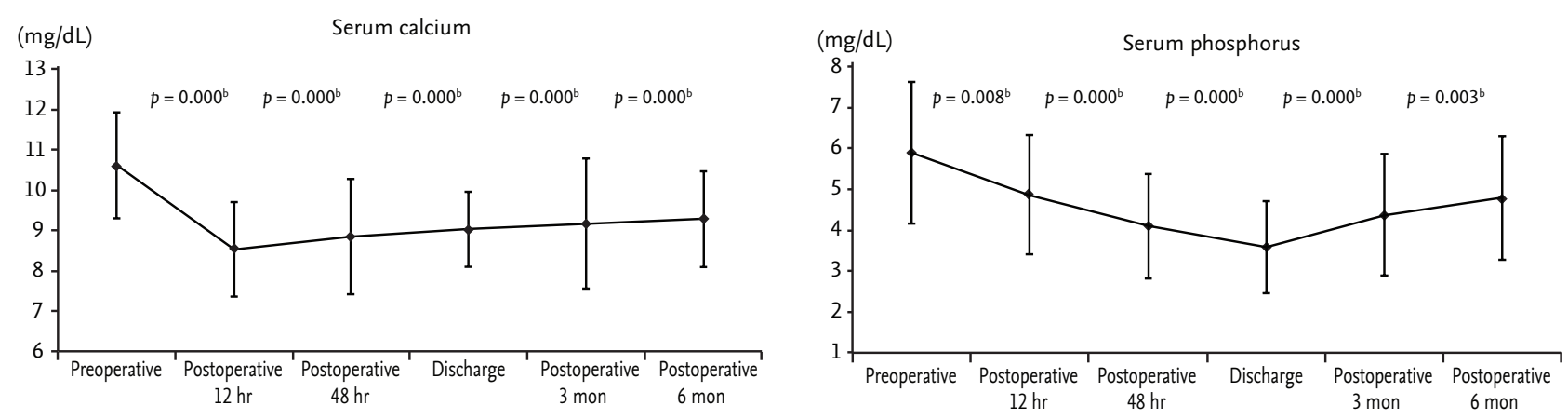

A

\section{B}
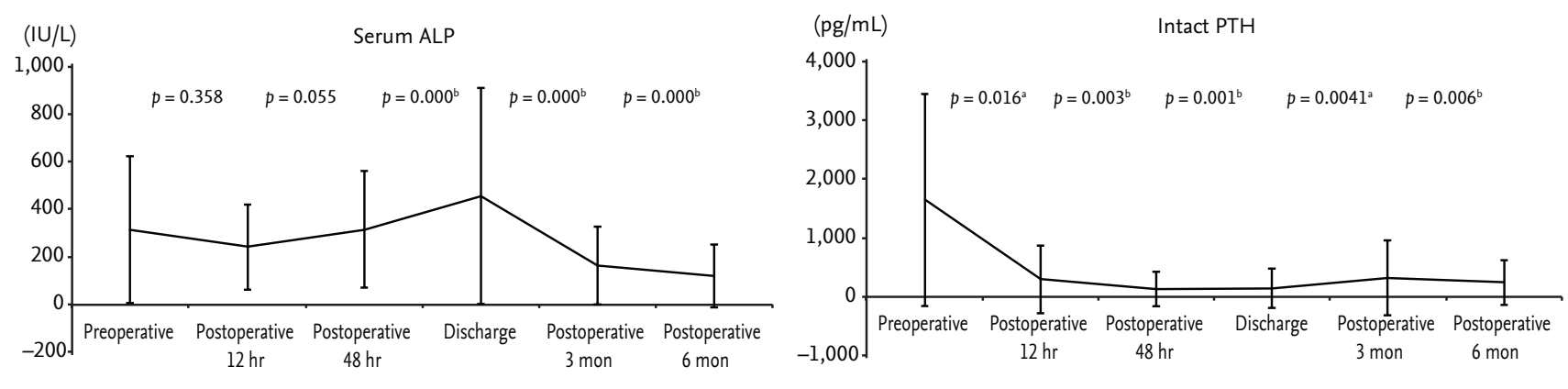

D

Figure 2. Changes in (A) serum calcium, (B) phosphorus, (C) alkaline phosphatase (ALP), and (D) parathyroid hormone (PTH) from preoperative levels and levels 12 hours postoperatively, 48 hours postoperatively, time of discharge, 3 months postoperatively, and 6 months postoperatively. All values are expressed as mean \pm SD. A $p<0.05$ was considered to indicate statistical significance (paired $t$ test). ${ }^{\mathrm{a}} p<0.05$ for comparison between the groups. ${ }^{\mathrm{b}} \mathrm{p}<0.01$ for comparison between the groups. 


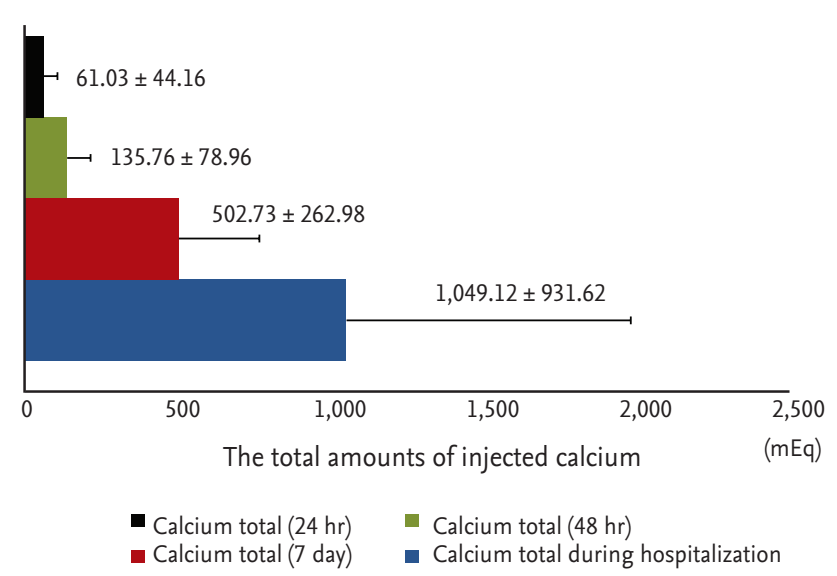

Figure 3. Total amount of calcium injected during 24, 48 hours, and 7 days after parathyroidectomy and during the total hospitalization period.

Table 2. Univariate analysis of total amount of injected calcium and parameters associated with bone and mineral metabolism by Pearson correlation analysis

\begin{tabular}{lcc}
\hline \multirow{2}{*}{ Variable } & \multicolumn{2}{c}{ Log } \\
\cline { 2 - 3 } (total amount of injected calcium) \\
\cline { 2 - 3 } Preoperative & \multicolumn{1}{c}{ p value } \\
\hline $\mathrm{Ca}$ & -0.063 & 0.560 \\
$\mathrm{P}$ & 0.118 & 0.278 \\
$\mathrm{ALP}$ & 0.453 & $0.000^{\mathrm{a}}$ \\
$\mathrm{PTH}$ & 0.275 & $0.010^{\mathrm{a}}$ \\
$\Delta \mathrm{Ca} 12 \mathrm{hr}$ & 0.249 & $0.020^{\mathrm{b}}$ \\
$\Delta \mathrm{P} 48 \mathrm{hr}$ & 0.346 & $0.001^{\mathrm{a}}$ \\
$\Delta \mathrm{ALP} 48 \mathrm{hr}$ & -0.011 & 0.921 \\
$\Delta \mathrm{Ca} 48 \mathrm{hr}$ & 0.051 & 0.639 \\
\hline
\end{tabular}

Ca, calcium; P, phosphorus; ALP, alkaline phosphatase; PTH, parathyroid hormone.

${ }^{a} p<0.01 .{ }^{b} p<0.05$.

Log (total calcium injected during hospitalization $)=$ $2.576+0.001 \times($ preoperative ALP $)+3.575 \times 10^{-5} \times($ preoperative $\mathrm{iPTH})+0.06 \times(\Delta$ phosphorus 48 hours $)$.

\section{DISCUSSION}

PTX remains one of the most important treatment modalities for secondary hyperparathyroidism refractory to medical treatment. This study showed our experience of PTX based on clinical characteristics and outcomes in a single center over 9 years. This study also showed the postoperative total calcium requirement and associated factors for total calcium requirements after PTX. Patients with primary hyperparathyroidism can be discharged immediately after PTX, whereas patients with secondary hyperparathyroidism on dialysis require much more postoperative calcium and a longer hospital stay [12]. Our results suggest a formula for the prediction of postoperative calcium required after PTX during hospitalization using significant variables from a multiple linear regression analysis.

We showed that in patients with poor cardiorespiratory reserve and refractory secondary hyperparathyroidism who were unlikely to be optimized adequately for GA, PTX under LA was an appropriate treatment option. In this study, a total of 11 patients had severe medical conditions such that they could not tolerate GA, and we performed limited PTX under LA. Though one patient died after limited PTX under LA, the cause of death was other medical problems. Recently, Cheong et al. [13] reported a series of total PTX under LA in patients with risk factors for GA. They enrolled 32 consecutive patients with severe cardiorespiratory dysfunction who underwent total PTX under LA over a period of 7 years. The postoperative complications were minimal and all patients reported symptomatic improvement [13]. Taken together, PTX can be performed safely and successfully under LA. Although PTX under LA is relatively safe and effective for high-risk patients, multiple PTX under LA is not yet considered a standard procedure for secondary hyperparathyroidism except in patients with very a high risk for GA. Multiple PTX under LA requires more precautions than cases performed under GA.

There is still much debate over the most efficient surgical technique in terms of avoiding both recurrence and the development of adynamic bone disease. Currently, subtotal PTX with a cervical remnant and total PTX with AT are standard procedures for secondary hyperparathyroidism [14,15]. In this study, a total PTX with $\mathrm{AT}$ in the forearm was the standard technique. In previous studies, the recurrence rate in total PTX with AT was reported as $10.5 \%$ (16 of 152 patients) [16]. In a series of 1,053 patients treated with total PTX with AT, the recurrence rate was $8.1 \%$ [8]. In contrast, the recurrence rate of total PTX without AT was reported as $4 \%$ (55 cases) [17] and $2.3 \%$ (43 cases) [18]. In this study, recurrence 
occurred in 10 of 59 cases (16.9\%) of total PTX with AT. No recurrence was found in total PTX without AT cases. In previous studies, subtotal PTX showed a higher recurrence rate (32.1\%) than total PTX with AT [19]. In the present study, the recurrence rates of subtotal PTX and limited PTX were 27.2\% (3/11cases) and 40\% (5/15 cases), respectively. Total PTX without AT showed the lowest recurrence rate, which was similar to previous studies for total PTX without AT and subtotal PTX.

A previous study explained a hypothesis for detectable PTH levels after PTX without AT as follows. During the embryological development of the parathyroid glands, it has been suggested that microscopic islands ("rests") of parathyroid tissue can be deposited anywhere along the descent of the glands from the third and fourth branchial pouches. Hyperplasia of these microscopic rests, attributable to the persistent uremia stimulus created by ongoing ESRD, may occur, resulting in detectable PTH in the long term despite total PTX without AT [20]. A recent study also proposed that total PTX without AT may still be considered the operation of choice in patients with aggressive forms of secondary hyperparathyroidism or of advanced dialysis vintage with no access to renal transplantation because of its low recurrence rate $[21,22]$. Theoretically, total PTX without AT may suit aggressive forms of secondary hyperparathyroidism more than a potential renal transplant in view of the increased frequency of very low or undetectable PTH in renal transplant recipients. However, this procedure may place them at greater risk of adynamic bone disease, compared with a dialysis-dependent population who do not undergo PTX. A prospective randomized trial and bone biopsies comparing total PTX without AT with both subtotal and total PTX with AT would clearly help to determine the incidence of adynamic bone disease.

Postoperative hypocalcemia or hungry bone syndrome is a common complication in patients with secondary hyperparathyroidism, but the management of hypocalcemia is not well defined in HD patients. Viaene et al. [23] reported transient hypocalcemia in more than $80 \%$ of patients after PTX despite frequent monitoring of the serum calcium level and adjustments of calcium and vitamin D supplements. Prolonged hypocalcemia despite continuous intravenous and oral calcium replacement increases hospitalization duration after PTX. From a clinical standpoint, the length of hospitalization after
PTX is significantly correlated with the grade of complications, and medical management and monitoring for postoperative hypocalcemia yield an increase in costs for the healthcare system. Thus, knowledge of the determinants of total calcium requirement after PTX is important for decreasing the length of hospitalization and improving calcium balance. Only a few studies have reported the total calcium requirement after PTX in patients with secondary hyperparathyroidism. Goh et al. [11] reported that ALP could predict calcium requirements after total PTX. They showed a significant correlation between serum ALP levels on admission and the elemental calcium dose at discharge, when corrected for serum calcium variation. In another study, a high preoperative PTH level and a low serum calcium level were identified as independent predictors of high postoperative calcium demands [23]. Cheng et al. [24] showed that a high preoperative ALP was significantly associated with a prolonged hospital stay in patients undergoing PTX for secondary hyperparathyroidism. In the present study, consistent with these reports, we observed a significant correlation with preoperative PTH, ALP, and the change in phosphorus during the first 48 hours postoperatively. We found statistically significant values that influenced the total injected calcium requirements during hospitalization (Table 2). The equation may be helpful for optimizing management strategies to prevent symptomatic hypocalcemia or prolonged hospital stays. Our center has managed postoperative hypocalcemia after PTX by means of implementation of a standardized protocol using specific calcium-oriented targets. Thus, our researchers are planning a further prospective randomized controlled study to reduce postoperative hospitalization duration after PTX by adjusting postoperative target calcium levels through application of this equation.

We acknowledge that our study has several limitations, mainly related to its retrospective nature. In addition, there was a relatively small sample size and short follow-up period. Thus, a larger study to confirm these findings is needed.

In conclusion, we propose PTX under LA as a treatment choice in patients with poor preoperative conditions. The standard surgical technique for PTX in secondary hyperparathyroidism is still controversial; our center decides the type of operation based on whether 
the patient is on the waiting list for kidney transplantation. Finally, preoperative ALP, preoperative iPTH, and $\Delta$ phosphorus at 48 hours postoperatively may be significant factors in estimating the duration of hospitalization and calcium requirements. A formula for the postoperative calcium requirement after PTX during hospitalization helps to prevent prolonged hypocalcemia by predicting the appropriate calcium requirement and to reduce the hospitalization period after PTX by adjusting postoperative target calcium levels.

\section{KEY MESSAGE}

1. In cases of poor cardiorespiratory reserve, if patients with refractory secondary hyperparathyroidism are unlikely to be optimized adequately for general anesthesia, parathyroidectomy (PTX) under local anesthesia is an appropriate option. The surgical technique with the lowest recurrent rate was total PTX without autotransplantation in the present study; however, this procedure may place patients with refractory hyperparathyroidism at a greater risk for adynamic bone disease, compared with a dialysis-dependent population.

2. Postoperative hypocalcemia is a very common complication after PTX. Prolonged hypocalcemia despite continuous intravenous and oral calcium replacement increased hospitalization duration after PTX. Preoperative alkaline phosphatase, preoperative intact parathyroid hormone, and $\Delta$ phosphorus at 48 hours may be significant factors that can be used to estimate postoperative calcium requirements after PTX.

3. A formula for the postoperative calcium requirement after PTX during hospitalization helps to prevent prolonged hypocalcemia by predicting the appropriate calcium requirement and to reduce the hospitalization period after PTX by adjusting postoperative target calcium levels.

\section{Conflict of interest}

No potential conflict of interest relevant to this article was reported.

\section{REFERENCES}

1. Glassford DM, Remmers AR Jr, Sarles HE, Lindley JD, Scurry MT, Fish JC. Hyperparathyroidism in the maintenance dialysis patient. Surg Gynecol Obstet 1976;142:328332.

2. Higgins RM, Richardson AJ, Ratcliffe PJ, Woods CG, Oliver DO, Morris PJ. Total parathyroidectomy alone or with autograft for renal hyperparathyroidism? Q J Med 1991;79:323-332.

3. Akizawa T, Fukagawa M, Koshikawa S, Kurokawa K. Recent progress in management of secondary hyperparathyroidism of chronic renal failure. Curr Opin Nephrol Hypertens 1993;2:558-565.

4. Hruska KA, Teitelbaum SL. Renal osteodystrophy. N Engl J Med 1995;333:166-174.

5. Yudd M, Llach F. Current medical management of secondary hyperparathyroidism. Am J Med Sci 2000;320:100106.

6. Stracke S, Keller F, Steinbach G, Henne-Bruns D, Wuerl P. Long-term outcome after total parathyroidectomy for the management of secondary hyperparathyroidism. Nephron Clin Pract 2009;111:c102-c109.

7. Tominaga Y, Numano M, Tanaka Y, Uchida K, Takagi H. Surgical treatment of renal hyperparathyroidism. Semin Surg Oncol 1997;13:87-96.

8. Tominaga Y, Uchida K, Haba T, et al. More than 1,000 cases of total parathyroidectomy with forearm autograft for renal hyperparathyroidism. Am J Kidney Dis 2001;38(4 Suppl 1):S168-S171.

9. Park JH, Kang SW, Jeong JJ, et al. Surgical treatment of tertiary hyperparathyroidism after renal transplantation: a 31-year experience in a single institution. Endocr J 2011;58:827-833.

10. Goldfarb M, Gondek SS, Lim SM, Farra JC, Nose V, Lew JI. Postoperative hungry bone syndrome in patients with secondary hyperparathyroidism of renal origin. World J Surg 2012;36:1314-1319.

11. Goh BL, Yudisthra MG, Hisham AN. Alkaline phosphatase predicts calcium requirements after total parathyroidectomy in patients receiving dialysis. Br J Surg 2010;97:185188.

12. Norman JG, Politz DE. Safety of immediate discharge after parathyroidectomy: a prospective study of 3,000 consecutive patients. Endocr Pract 2007;13:105-113.

13. Cheong YT, Taib NA, Normayah K, Hisham AN. Total 
parathyroidectomy under local anaesthesia for renal hyperparathyroidism. Asian J Surg 2009;32:51-54.

14. Schlosser K, Veit JA, Witte S, et al. Comparison of total parathyroidectomy without autotransplantation and without thymectomy versus total parathyroidectomy with autotransplantation and with thymectomy for secondary hyperparathyroidism: TOPAR PILOT-Trial. Trials 2007;8:22.

15. Melck AL, Carty SE, Seethala RR, et al. Recurrent hyperparathyroidism and forearm parathyromatosis after total parathyroidectomy. Surgery 2010;148:867-873.

16. Henry JF, Denizot A, Audiffret J, France G. Results of reoperations for persistent or recurrent secondary hyperparathyroidism in hemodialysis patients. World J Surg 1990;14:303-306.

17. Saunders RN, Karoo R, Metcalfe MS, Nicholson ML. Four gland parathyroidectomy without reimplantation in patients with chronic renal failure. Postgrad Med J 2005;81:255-258.

18. Ljutić D, Cameron JS, Ogg CS, Turner C, Hicks JA, Owen WJ. Long-term follow-up after total parathyroidectomy without parathyroid reimplantation in chronic renal failure. QJM 1994;87:685-692.

19. Hargrove GM, Pasieka JL, Hanley DA, Murphy MB. Short- and long-term outcome of total parathyroidectomy with immediate autografting versus subtotal parathyroidectomy in patients with end-stage renal disease. Am J Nephrol 1999;19:559-564.

20. Farrington K, Varghese Z, Chan MK, et al. How complete is a total parathyroidectomy in uraemia? Br Med J (Clin Res Ed) 1987;294:743.

21. Conzo G, Perna AF, Sinisi AA, et al. Total parathyroidectomy without autotransplantation in the surgical treatment of secondary hyperparathyroidism of chronic kidney disease. J Endocrinol Invest 2012;35:8-13.

22. Puccini M, Carpi A, Cupisti A, et al. Total parathyroidectomy without autotransplantation for the treatment of secondary hyperparathyroidism associated with chronic kidney disease: clinical and laboratory long-term follow-up. Biomed Pharmacother 2010;64:359-362.

23. Viaene L, Evenepoel P, Bammens B, Claes K, Kuypers D, Vanrenterghem Y. Calcium requirements after parathyroidectomy in patients with refractory secondary hyperparathyroidism. Nephron Clin Pract 2008;110:c80-c85.

24. Cheng SP, Liu CL, Chen HH, Lee JJ, Liu TP, Yang TL. Prolonged hospital stay after parathyroidectomy for secondary hyperparathyroidism. World J Surg 2009;33:72-79. 